Journal of Transport and Land Use 1:2 (Fall 2008) pp. 121-151

Available at http://jtlu.org

\title{
The role of employment subcenters in residential location decisions
}

\author{
Eun Joo Cho, Daniel A. Rodriguez, Yan Song \\ Department of City and Regional Planning \\ University of North Carolina Chapel Hill
}

\begin{abstract}
In this paper we employ Mecklenburg County, North Carolina, a polycentric city with 10 employment subcenters, as a case study to explore the role of employment subcenters in determining residential location decisions. We estimate discrete choice models of residential location decisions: conditional logit models and heteroscedastic logit models with both the full choice set and sampled choices. We find that access to certain employment subcenters, measured in terms of generalized cost, is an important determinant of households' residential location decisions. The proximity to specific employment subcenters varies across households with different income levels. These patterns can be explained by existing land use and transportation patterns, as well as by subcenters' economic specialization.
\end{abstract}

Keywords: Polycentric cities; Employment subcenters; Residential location; Logit models.

\section{Introduction}

Accessibility continues at the forefront of discussions regarding the integration of transportation and land development. Transportation investments enhance accessibility, thereby contributing to the possibility of additional development. Similarly, changes in land development, such as bringing destinations closer to origins, also enhance accessibility. Among the different destinations that can be accessed, access to workplaces has long been examined in studies of travel behavior. Voluminous research has examined criss-cross commuting patterns in polycentric cites. Much of the previous research has assumed that commuting time and cost are endogenous to people's decisions about where to live and work. Thus, a renewed emphasis on location decisions is critical to examining the importance of accessibility for transportation and land development.

To date, the preponderance of research on residential location decision-making has assumed that work locations are exogenously determined (Abraham and Hunt 1997; Levine 1998; Clark et al. 2003)—-that is, that workplaces are identified independently

${ }^{a}$ Daniel A. Rodriguez: danrod@email.unc.edu; Yan Song: ys@email.unc.edu

Copyright 2008 Eun Joo Cho, Daniel A. Rodriguez, and Yan Song.

Licensed under the Creative Commons Attribution - NonCommercial License 3.0. 
before residential locations are selected. Some researchers (Waddell 1993; Dietz 1998; Romani 2003) have aptly questioned the validity of this assumption and developed joint workplace and residence location models. Increases in job mobility, the prevalence of two-worker households, and the decentralization of urban areas suggest that accessibility to potential employment or activity centers may be a dominant determinant in explaining location choices. Crane (1996) showed that individuals consider both current job sites and potential job sites when evaluating their residential location and commute length, and that uncertainty about potential future job sites increases commute length. Thus, for location decision-making, actual work access may matter less than access to potential work locations. Additionally, the location of other urban functions that are frequently co-located with employment sites, such as retail stores, may contribute to the relevance of potential work sites for location decisions.

This study attempts to further analyze residential location decisions and accessibility to employment subcenters. A high-density employment cluster that influences urban structure and the Central Business District (CBD) is regarded as a major subcenter. It contains employment and activity opportunities. Specifically, this study aims to examine how accessibility to subcenters influences residential location decisions in Mecklenburg County, North Carolina, while controlling for household characteristics and for potential residential locations' built-environment characteristics. Workplace choice is assumed to be endogenous to residential location decision-making, and employment subcenters are assumed to be potential workplaces as well as activity centers for both workers and non-workers (some of whom may be searching for jobs currently or in the future). Thus, this work contributes to the literature by extending the scope of the analysis of access to the workplace to include all households. Two additional contributions made in this study are the exploration of various forms of interrelationship among employment and activity centers from substitutes to complements, and the development of an access measure that includes multimodal transportation and non-motorized means of transportation.

The next section summarizes the literature on residential location decisions, and is followed by sections on methods, study area, results and their discussion, and a conclusion.

\section{Literature review}

This research is related to a large body of studies determining factors of residential choice behavior of households. The intention here is to highlight areas where existing literature most closely intersects this research, rather than to review the residential choice literature comprehensively. This review focuses two bodies of literature relevant to our effort: the relationship between polycentric urban structure and commuting time, and the development of measures of accessibility to employment. 
Studies have examined whether households commute less in polycentric cities. Research findings have suggested that polycentric cities generate "wasteful" or "excess" commuting (Hamilton 1982; Small and Song 1992), although this appears to result from skill specialization and measurement error (Rodríguez 2004). On the other hand, other evidence shows that in polycentric cities, the proximity between jobs and houses results in lower commuting times and shorter commute distances (Dubin 1991; Gordon et al. 1991; Giuliano and Small 1991; Cervero and Wu 1997; Clark et al. 2003). For example, Dubin (1991) shows that job suburbanization shortens commuting time. Giuliano and Small (1999) and Cervero and Wu (1997) find that the average commute length and time of workers in suburban employment centers are shorter than those of workers in urban centers, which suggests that commute distance and time impedance are strong determinants of residential location choice for workers in suburban centers. Levinson (1998), in an analysis of job and accessibility gradients around the CBD in the Washington, D.C. area, also shows that downtown and suburban employment centers attract commuters to live close by.

Studies on home and work locations in polycentric cities have also been conducted across industry sectors (Dubin 1991; Crane and Chatman 2003; Romani 2003). Dubin (1991) reveals that workers in sales and service have shorter commuting times due to firms' decentralization. However, Crane and Chatman (2003) show that suburbanization of retail and service employment is not strongly associated with commute length, but that other sectors such as construction and wholesale are associated with shorter commutes, and manufacturing and finance are associated with longer commutes. This is consistent with the view that industry sectors benefiting from agglomeration economies (like finance and banking) are less likely to decentralize, whereas sectors like retail and manufacturing have decentralized following people (Glaeser and Kahn 2001), and therefore that shorter commutes are expected for workers in decentralized economic sectors. However, Romani (2003) argues that shorter commuting probability is higher in banking and finance industries than in retail and other sectors related to food and accommodations. In summary, these studies exhibit inconsistent results, which can be explained by the fact that the decentralization of industry is affected by spatial and temporal variations and thus proceeds at different paces and takes different forms. Although polycentricity provides opportunities for shorter commuting times, actual commuting times are likely to depend on industry sector and skill specialization, and on characteristics of the local housing market.

Another body of research relevant to the current application deals with various methods of measuring accessibility. Baradaran and Ramjerdi (2001) identify five different types of accessibility measures. El-Geneidy and Levinson (2006) provide a detailed review of the literature on accessibility measures, which are described very briefly below. Earlier measures of accessibility account for travel time or distance or cost between origin and destination. More recently, the cumulative opportunity mea- 
sure counts the number of potential opportunities (such as the number of firms or the number of recreational opportunities) that can be reached within a predetermined travel time (or distance). This measure, not accounting for the size of the facility or the impedance of reaching it (cost) and making artificial decisions on the predetermined travel time or buffer distance, is simple to compute and understand. For example, Bhat and Guo's (2004) accessibility measures consider opportunities to access both jobs and socio-recreational or retail sites; the researchers find that households prefer locations that provide greater accessibility to shopping.

Gravity-based accessibility measures are the most widely used methods of measuring accessibility. These measures are expected to increase with the increase in the activity opportunities nearby but to decline the farther (in terms of time, distance, or generalized cost) the opportunities are from the origin. In order to compute a gravity-based measure, considerable effort is needed to develop an impedance factor and to determine the appropriate weighting factors for the destination. Utility-based measures are related to gravity-based measures, and are used to incorporate variations in individual traveler preferences and to account for human choice by including the attractiveness of each destination based on economic benefits that people derive from having access to different destinations. Computation of a utility-based measure is data intensive. Constraints-based or people-based measures (Wu and Miller 2002) take the amount of time available into consideration. Finally, composite accessibility (Miller 1999) combines space-time and utility-based approaches into one measure.

Using one or more of these accessibility measures, previous studies have evaluated how accessibility affects housing location choice. For examples, variables capturing job accessibility (Anas 1981, 1982, 1995; Ben-Akiva and Bowman 1998) have been included in choice models and most have been found to be statistically significant. Using data from Calgary (Canada), Abraham and Hunt (1997) found that distance-related variables (journey-to-work out-of-pocket costs and trip time) are the most important location factors influencing residential choices. Similarly, in a study that explicitly addressed the importance of commuting variables, Levine (1998) estimated a nested logit model for the Minneapolis-St. Paul area and found that commuting time is the variable with higher relative importance compared to community characteristics such as school quality, taxes, and crime rate. Levinson (1998) also pointed out the relative importance of accessibility, showing that accessibility to jobs and housing are more effective variables than demographic and socio-economic variables such as age, gender, home ownership, number of children and household size. Shen (1998) and Bhat and Guo (2004) also confirmed that accessibility to the workplace is a critical determinant of residential location choice. Zondag and Pieters (2005) showed that people in the Netherlands are less likely to move to locations with less accessibility for all purposes. Some other studies, however, have found that accessibility is not an influential factor in residential location decision; Giuliano and Small (1993), for example, argued that 
accessibility to workplaces only slightly influences residential location decisions.

In considering accessibility, relatively little attention has been paid to the explicit identification of employment and activity subcenters. A focus on subcenters is relevant because, although the majority of jobs are now found outside of established centers, it is the concentration of jobs that characterizes employment and activity subcenters. As highlighted by Shen (2000), from an individual's perspective, the concentration of jobs in subcenters is important for job-seeking and job-finding. For firms, the concentration of activity has tangible impacts on location decisions due to agglomeration economies (McCann 2001; Shukla and Waddell 1991). A focus on subcenters is also important because of public transportation's economies of densities, which benefit from the spatial concentration of activity.

In addition to accessibility, additional factors have been tested in models of households' location decisions such as housing demand models (Rapaport 1997; Reginster and Nagot 2005; Cho et al. 2005) and discrete choice models (McFadden 1978; Nechyba and Strauss 1998; Sermons and Koppleman 1998; Molin and Timmermans 2003; Zondag and Pieters 2005). Housing attributes such as physical characteristics, ownership and type of dwelling are also often relevant. More recent residential location studies include various variables involving neighborhood and public service characteristics: neighborhood environmental amenities, community ethnicity, density, school quality, crime rates and different measures of public service provision and accessibility. These variables significantly influence residential location choice.

Socio-demographic characteristics such as income, presence of children, ethnicity, occupational status and private automobile availability have been found to strongly determine residential location choice. Accessibility measures have been differentiated by socio-demographic characteristics, like multiple-worker households relative to singleworker households (Waddell 1996; Abraham and Hunt 1997; Sermons and Koppelman 1998; van Ommeren et al. 1998; Clark et al. 2003; Plaut 2006; Mok 2007), for males versus females (Waddell 1996; Abraham and Hunt 1997; Sermons and Koppelman 1998, 2001; Clark et al. 2003; Plaut 2006; Mok 2007), and for occupational class (Deitz 1998; Clark et al. 2003; Mok 2007). These studies claimed that assuming single-worker households and using aggregated information may lead to faulty conclusions about the influence of access to workplaces on residential location decisions. In contrast to Levinson (1998), Molin and Timmermans (2003) and Zongag and Pieters (2005) conclude that demographic characteristics, housing attributes and neighborhood attributes are more influential factors than accessibility in residential location decision-making.

In summary, the literature suggests that polycentricity offers opportunities for commuting savings. Whether such reductions materialize is a matter of empirical debate. At the same time, the consideration of access to destinations in polycentric areas is varied, with limited regard for employment concentrations for residential loca- 
tion decision-making. In this study, we explore how the accessibility to employment subcenters contributes to explaining residential location decisions. We measure access to employment by using a generalized cost function from a multimodal (motorized and non-motorized) mode choice model. In addition, we explore whether households with different income levels have different preferences regarding proximity to different employment subcenters and whether subcenters interact, by either competing with or complementing one another, in the eyes of residential location decision-makers.

\section{Methods}

To analyze the effects of accessibility to employment subcenters on residential location choices, this study relied on random utility theory to estimate discrete choice models of residential location decision-making. This theory is based on the assumption that rational people choose residential locations that maximize their utilities. This study also assumed that every household moves freely to any residential location without any constraint on housing availability. Thus, the residential location choice models used in this work do not reflect housing supply and availability.

\subsection{Study area}

Mecklenburg County, North Carolina, was chosen as an example of a polycentric city. Charlotte, the main city in Mecklenburg County, has been one of the fastest-growing metropolitan regions in the United States since the Second World War. The city has experienced a rapid increase in population, with 22 percent growth from 1990 to 2000 (US Census), and its population reached approximately 610,949 in 2005. If current trends continue, Charlotte and its sphere of influence are expected to experience a population increase of 330,000 new residents between 2005 and 2030.

However, population density in Charlotte has decreased from 6.98 persons per acre in 1950 to 3.60 per acre in 2000. This indicates that land consumption has increased even faster than population growth. As shown in Figure 1, Charlotte did not have large, dense residential cores in 2000. Only a few scattered zones currently have more than six persons per acre, and the mean population density is approximately three persons per acre. This urban spatial structure has resulted in a scattering of residences throughout the entire metropolitan area, fueling land consumption, decreasing air quality and increasing traffic congestion.

As expected, employment growth has gone hand-in-hand with population growth. By 1980, manufacturing related to textiles was the main industry in the city. While most of the manufacturing industry has since disappeared, Charlotte rapidly became one of the world's leading banking centers following the arrival of Bank of America and Wachovia in 1951 (Smith 2004). In a short time, Charlotte has rapidly established a 
Figure 1: Population density in Mecklenburg County.

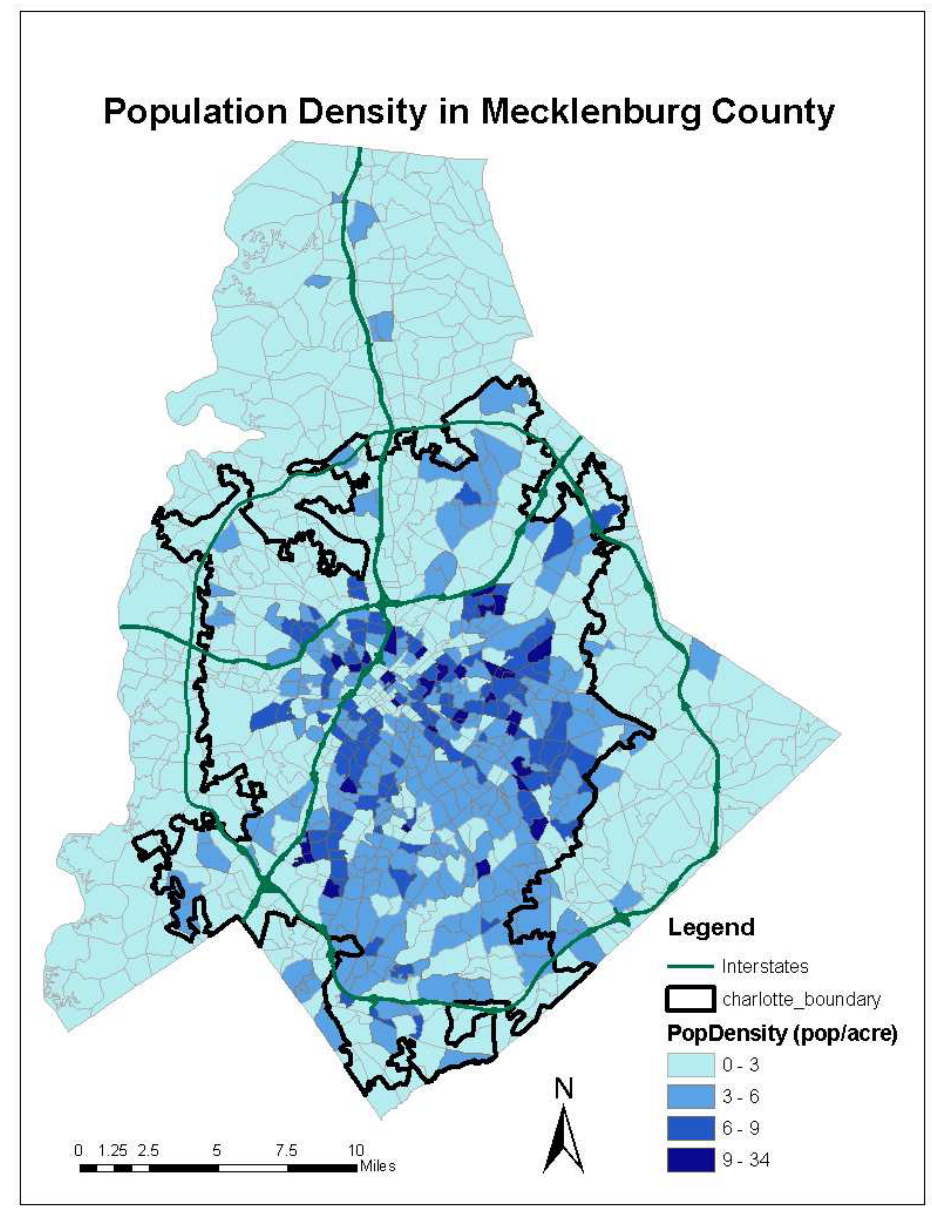

diverse economic base, including industries such as financial services, various other services, retail and wholesale trade, manufacturing, and government. With this rapid economic development, numerous new job opportunities in various business sectors have been generated and have been spread over all the employment subcenters in the county.

\subsection{Data}

This study is based on two major data sources: 1) household-level demographic and travel data; and 2) zonal demographic, employment and built-environment data. House- 
hold-level demographic and travel data were obtained from the 2002 Greater Charlotte Region Household Travel Survey, which was administered by the area's metropolitan planning organization (MPO). Surveyed households were selected using List-Assisted Random-Digital-Dialing from the directory-listed phone numbers. A total of 1,510 households in Mecklenburg County were sampled. The households completed travel diaries and provided 24-hour trip information during weekdays between January 13 and May 7, 2002.

Zonal data were derived from 1,024 traffic analysis zones (TAZs) defined by the MPO as being located within Mecklenburg County. Zonal demographic and employment data were obtained from the 2000 Census Transportation Planning Package (CTPP), and zonal spatial data characterizing the built environment were provided by the MPO, county planners and the Charlotte Department of Transportation.

Independent variables in the residential location models included: access to employment subcenters; seven neighborhood typologies defined using cluster analysis; two factors measuring walkability and local accessibility to various land uses and transit systems; and other demographic and zonal indicators. Because the study area was limited to a single county, it was assumed that taxes, school districts, and other local public goods were similar across the study area.

\subsection{Defining employment subcenters}

Many studies have defined employment subcenters using a variety of methods such as employment density statistical functions, employment and population size, and minimum-density thresholds (McDonald 1987; Giuliano and Small 1991, 1993; Cervero and Wu 1997; McMillen and McDonald 1998; McMillen 2001; Craig and Ng 2001). However, none of these methods can explain well the formation and growth of employment subcenters in all metropolitan areas. Moreover, different methods generate various numbers, sizes and boundaries of employment subcenters, even in the same area.

Among these methods, the minimum-density thresholds approach suggested by Giuliano and Small (1991) has been chosen by many other researchers (Cervero and Wu 1997; McMillen and McDonald 1998) for its efficiency. Giuliano and Small (1991) defined 28 employment subcenters in the five-county Los Angeles (LA) region by identifying a contiguous set of zones, each with 10 employees per acre and with a combined minimum of 10,000 employees. In later work (1999), the same researchers also identified 33 employment subcenters when they reduced the minimum employment to 3,000 employees. It is notable that the number of subcenters and their locations change according to the threshold selected.

Giuliano and Small's method advantageously provides a flexible way to identify employment subcenters in a particular area through thresholds set by analysts who 
are familiar with the area under consideration. For that reason, their method was adopted to define employment subcenters in Mecklenburg County based on employment density and size. Because Mecklenburg County is a much smaller metropolitan area than the Los Angeles (LA) region in terms of population (6.58\% of the LA region), size (1.55\%), and employment (10.35\%), minimum thresholds were lowered based on local knowledge, focusing primarily on grouping diverse, dense employmentbased TAZs into several employment clusters. These robust thresholds could capture potential growing employment subcenters in the county.

We performed a sensitivity analysis to Giuliano and Small's criteria by applying different minimum thresholds. When we applied the original criteria (10 employment density and 10,000 combined employment), only two employment subcenters were identified as accounting for $21.7 \%$ of the county's total employment ${ }^{1}$ It is not surprising that the original criteria were too rigorous (high) to effectively describe a metropolitan area that is so much smaller than the Los Angeles, Chicago, or San Francisco Bay areas to which they had been applied in previous studies.

Using minimum thresholds of four employees per acre (the mean employment density in the county) and a combined minimum of 5,000 employees identified a total of 10 employment subcenters, including the CBD. These employment subcenters offered 257,460 jobs out of a total of 474,971 jobs in the county, accounting for 54.2 percent of total employment. These minimum thresholds met the goal of simplifying urban spatial patterns of employment clusters so as to capture more than half of the county's total employment. Furthermore, the locations of the 10 employment subcenters match up well with the transit hubs of the county's 2025 system plan, which are and will be the focus of employment and activities. Therefore, these 10 employment subcenters were subjectively selected as the best choice in the study area (Figure 2).

The specialization of individual employment subcenters was also examined using the location quotients (LQ) technique. A total of 12 business sectors (identified by twodigit North America Industry Classification System (NAICS) codes) appear to have a significant presence in Mecklenburg County. Among them, nine business sectors have concentrations in at least one employment subcenter. Subcenter 1, located at the north of the CBD, specializes only in professional services. On the contrary, most of the employment subcenters specialize in numerous similar business sectors: arts, professional and financial services, various other services and transport/warehouse/utilities. With these sectors' dispersion pattern, employment subcenters are expected to grow with similar industry mixes. This implies that employment subcenters in Mecklenburg County generally continue to provide similar jobs and services to households. Con-

\footnotetext{
1 We defined employment subcenters as a connected set of TAZs, each with a minimum employment density threshold and a combined minimum employment threshold, rather than a contiguous set of zones, as suggested by Giuliano and Small (1991).
} 
Figure 2: Employment subcenters in Mecklenburg County.

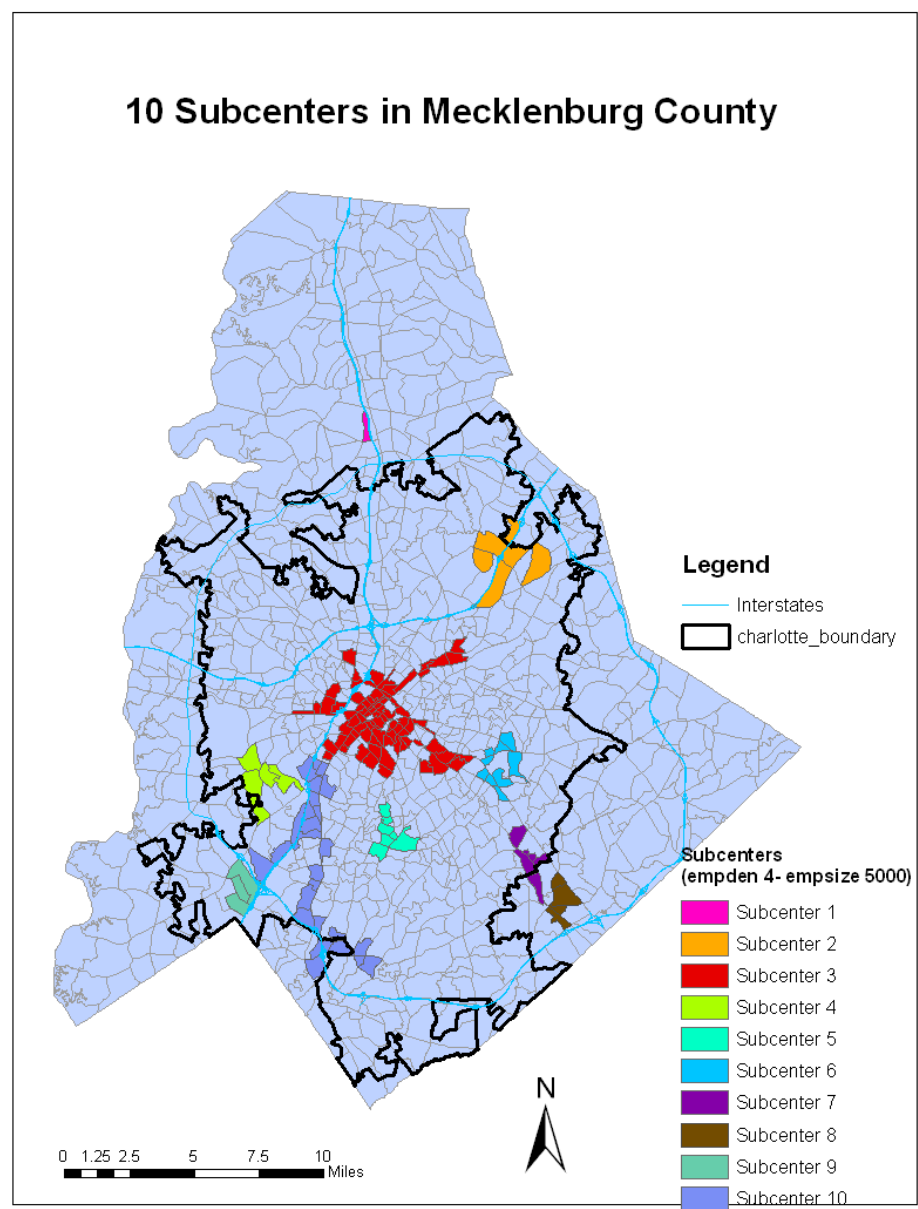

sequently, easy accessibility to various employment and activities may expand households' potential residential location choices if the housing market supplies diverse housing options near employment subcenters. On the other hand, retail, manufacturing and educational/health/social services are not significantly specialized in employment subcenters. These sectors must be widely dispersed without being affected by agglomeration economies.

Table 1 shows the employment characteristics of each subcenter. Two employment subcenters (subcenters 3 and 10) are the foci of economic growth in the county, accounting for 65.0 percent of the 10 subcenters' total employment. Subcenter 3 
comprises the CBD, and subcenter 10 is formed along an interstate highway. This formation of employment subcenters is similar to that seen in other studies (Giuliano and Small 1991; Cervero and Wu 1997), which found that the CBD subcenter has the greatest influence on economic growth among other employment subcenters and that a subcenter located along a highway corridor is also the focus of employment. The latter finding indicates that existing transportation facilities stimulate economic growth by attracting more jobs. Based on these findings, we determined that employment in Mecklenburg County is mainly clustered in the CBD and along a highway corridor, and that the other subcenters are thus functionally connected to the CBD.

\subsection{Measuring access to subcenters}

To borrow Axhausen's (2007) definitions of access and accessibility, accessibility to employment subcenters is regarded as a composite good measuring the total quality of access to a set of employment subcenters with trade-offs allowed. In this context, the accessibility of employment subcenters can be studied by understanding whether and how access to employment subcenters matters in residential location choices.

Access to individual employment subcenters was measured using the log-sum denominator of a mode choice model estimated for Mecklenburg County. This mode choice model is multimodal, so the generalized cost of accessing a given employment subcenter will account for the various transportation opportunities available from particular locations that influence residential location choice. Log-sum accessibility measures have been proposed and applied in other studies (Niemeier 1997; Ben-Akiva and Bowman 1998; Berechman and Paaswell 2001; Berke et al. 2006 pp. 245-246) but the data requirements of this approach have limited its popularity in the U.S. (Handy and Clifton 2001). Mode choice coefficients have been used frequently as accessibility measures in combined land use and transportation models developed in some European countries; for example, the DELTA model developed by Simmonds during 1995-1996 in the UK (Timmermans 2003) and the TIGRIS XL model package developed for the Transportation Research Centre in the Netherlands (Zondag and Pieters 2005).

The mode choice model used in this study is distinct from previous applicatins, however, in that it includes non-motorized options (walking and bicycling) and thus appropriately represents transportation accessibility in space. In addition to accounting for in-vehicle and out-of-vehicle travel time, travel cost, and individual-level characteristics including income, gender, age, and vehicle availability, the mode choice model also accounts for the impact of the built environment on the probability of non-motorized travel, as suggested by Rodríguez and Joo (2004) and Cervero (2002).

Once the generalized cost of each employment subcenter had been identified, the accessibility of the employment subcenters was modeled using three different ap- 


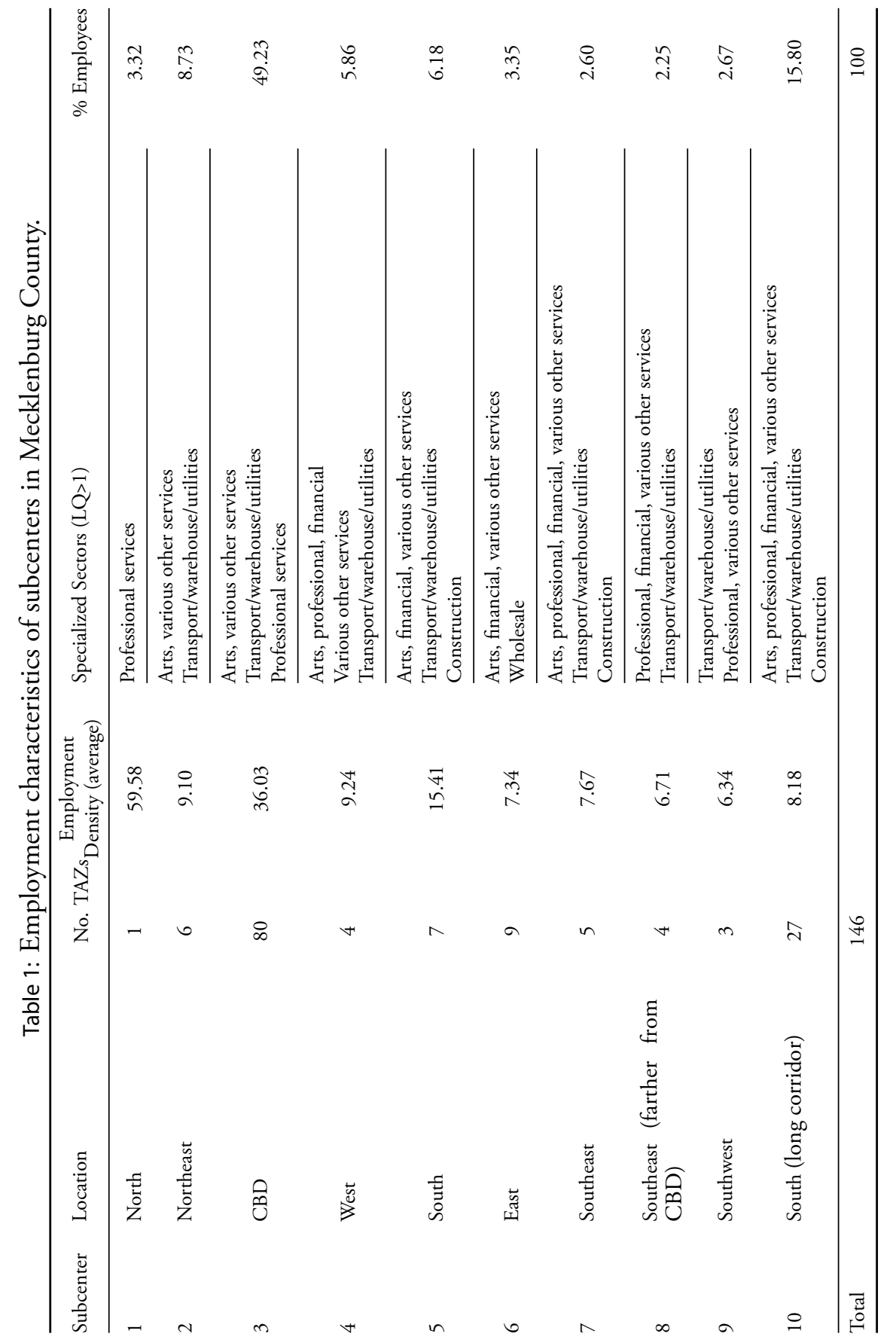


proaches, built on suggestions by Anas, Small and Arnott (1998), in order to examine how the employment subcenters interact from the perspective of households. These three different access models included:

1. Access to all and each subcenter individually as separate independent variables in the residential choice model, assuming that households consider access to all individual subcenters in their location decision-making and value each subcenter differently;

2. Access to the closest subcenter from each TAZ, based on the assumption that subcenters can be substitutes - that is, households prefer to live close to the closest subcenter among others; and,

3. The cumulative sum of accessing each employment subcenter from each TAZ, based on the assumption that subcenters can complement one another-that is, households consider all access to each subcenter together and choose a location with higher accumulative accessibility to all subcenters.

The effects of accessibility to employment subcenters on residential location decision were examined by comparing the results of these three approaches.

\subsection{The built environment of each TAZ}

This study employed a neighborhood typology and built-environment measures already developed in the context of a related research grant. The neighborhood typology was derived from a cluster analysis of five built-environment factors that describe walkability, accessibility, agglomeration, property value, and industry. A total of 373 block groups in our study area were reduced to eight neighborhood types, as shown in Figure 3 (aggregated TAZs). The resulting typology was vetted by local planners, who helped increase the face validity of the results. For this study,the first and second neighborhood types were combined, because no households reside in the core CBD. The final eight neighborhood types, shown in Figure 3, were:

- 1 = core $\mathrm{CBD}$,

- 2 = CBD with residence,

- 3 = first ring of suburbs,

- 4 = second ring of suburbs,

- 5 = suburban single family residences with some commercial and transit services,

- 6 = suburbs with low regional access, 
- 7 = exurbs with low regional access,

- $8=$ isolated residences in forested, industrial and commercial areas.

Figure 3: Neighborhood types in Mecklenburg County.

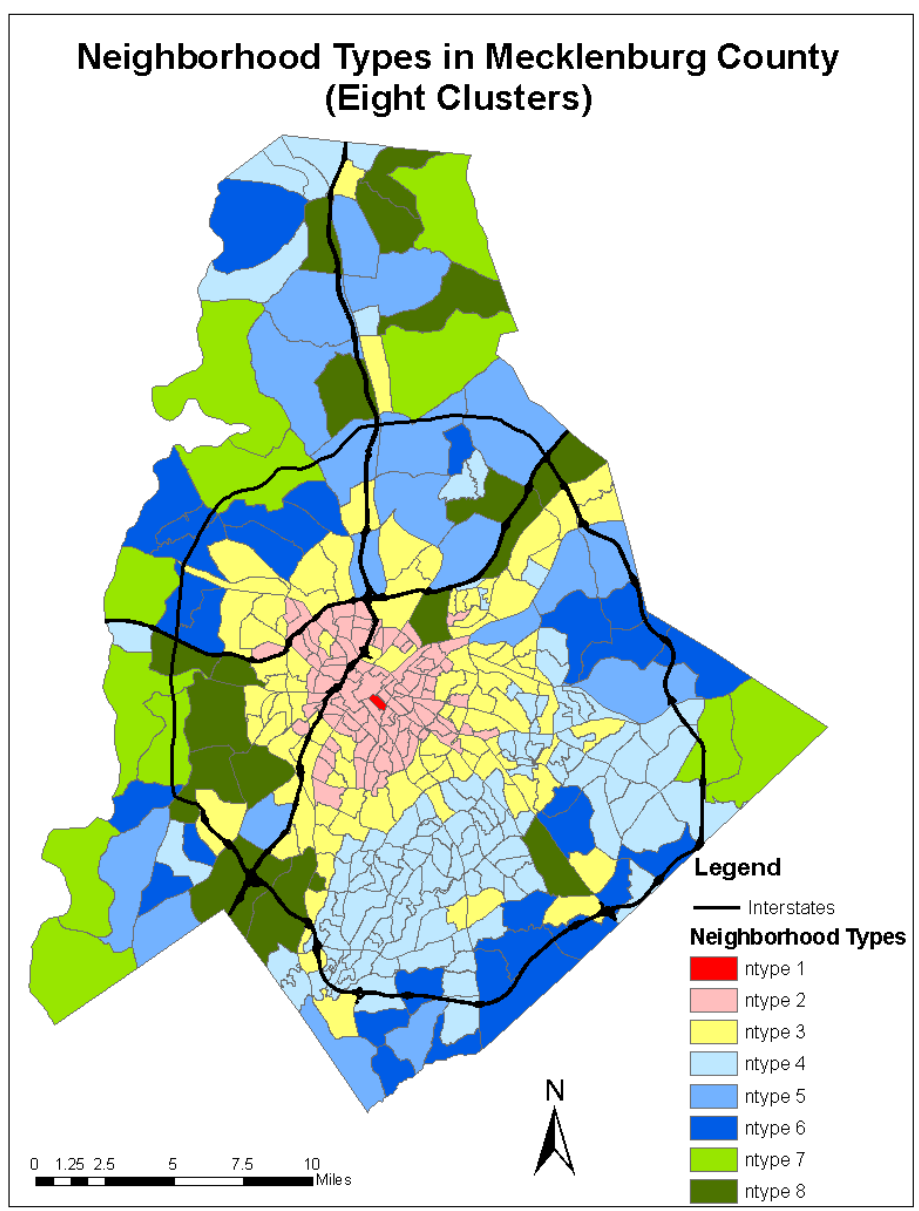

In addition to the typology, two built environment factors were used to characterize each TAZ: walkability and local accessibility. These two factors were derived through exploratory factor analysis by combining several observed indicators. Other studies indicated that the impact of a single built environment attribute may be too small to detect; however, when the built environment attributes that cause a similar impact on residential location choice are combined, these impacts become cumulative and 
may be large enough to be detected, with a synergistic effect generated by additional interaction terms of built environment attributes influencing decision-makers.

\subsection{Model specification}

The measures of access to subcenters and the built-environment measures were incorporated into a cross-sectional analysis with an estimation of two types of discrete choice models of residential location decision: a conditional logit model and a heteroscedastic logit model. Each model type was estimated for three measures of accessibility to employment subcenters (access to each, access to closest subcenter, and cumulative access to all subcenters) for three income groups (low, medium, and high) ${ }^{2}$ on the grounds that income is likely a surrogate for different tastes and location preferences. Furthermore, these models were estimated assuming both that all TAZs are available to decision-makers, and assuming that only a reduced sample of them is available. In total, 48 models were estimated (two types of models, three access specifications, three income levels and overall, two choice options - full or partial). The models were then compared to each other in order to determine which among them exhibited the best fit, and this subset is reported in this article.

To account for the one-level conditional logit model's limitations, we estimated a one-level heteroscedastic logit model. In response to questions about the validity of the homogeneous error terms of the multinomial logit model, several ways to deal with heteroscedasticity of the unobserved effects have been proposed and tested in many applications (Allenby and Ginter 1995; Bhat 1995; Hensher et al. 1999; DeShanzo and Fermo 2002; Hole 2006). These models account for heteroscedasticity across alternatives and/or individuals. Among these models, we used a heteroscedastic logit ${ }^{3}$ model that allows error variance to vary across households by specifying random effects of scale parameters. Thus, in our heteroscedastic logit model, choice probabilities are given by

$$
P_{i j}=\frac{\exp \left(\mu_{i} V_{i j}\right)}{\sum_{k=1}^{m} \exp \left(\mu_{i} V_{i k}\right)}
$$

where $V_{i j}$ is a vector of attributes of the $j$ th alternative perceived by the $i$ th household and $\mu_{i}$ is a scale parameter that is a function of $i$ th household characteristics and is thus constant across choices. Household characteristics considered in our models are

\footnotetext{
${ }^{2}$ Low-income households were defined as those earning less than $\$ 30,000$ per year, medium-income households as those earning $\$ 30,000$ to $\$ 75,000$ per year, and high-income households as those earning more than $\$ 75,000$ per year.

${ }^{3}$ We estimated the heteroscedastic logit model by using the STATA software program with the command of clogithet.

${ }^{4} \mu_{i}$ is parameterized as $\exp \left(Z_{i} \psi\right)$, where $Z_{i}$ is a vector of household characteristics and $\psi$ is a vector of parameters that capture the effect of household characteristics on the error variance.
} 
number of children, income, number of motorized vehicles and number of workers in a household. Of interest to this study, the utility of each alternative (TAZ level) was modeled as a function of several elements: access to subcenters, land devoted to highincome residences, percentage of black residents, neighborhood types, walkability, and accessibility to various land uses in each TAZ.

In the models, the choice being modeled is that of a TAZ, with the full choice set composed of the 1,024 TAZs. Using such a large choice set is controversial for theoretical and empirical reasons. One of the main reasons is that the independence from irrelevant alternatives (IIA) assumption among TAZs rarely holds. Another reason is that, as Lerman (1975) pointed out, the introduction of clearly unavailable choices into an individual's choice set may lead to biased estimates.

Based on the well-known result that a sample of choices results in consistent estimates of the multinomial logit model (McFadden 1978), two approaches were used to reduce the choice set: a random sampling approach containing a sample of non-chosen TAZs and a chosen TAZ for each household (Miller et al. 2004); and an approach that includes both a deterministic and a random component.

For the deterministic-and-random approach, housing affordability served as a deterministic rule to reduce the choice set, based on the suggestion by Levine (1998) and others that housing affordability constrains residential location choice for households. In this way, only TAZs within a housing affordability threshold (defined as the ratio of the median home price in the county to the median household income of each respondent) were included. This financially available choice set may lead to more consistent and unbiased estimates and to smaller standard errors than the universal choice set (that also includes financially-infeasible choices). After applying the home price multiplier as a deterministic rule, small choices were randomly sampled to deal with complexity. The result was a choice set that varies across households based on their income; the number of TAZs available to a household ranged from a minimum of 18 to a maximum of 43 .

\section{Results}

Table 2 shows descriptive statistics for TAZ and household characteristics. Among 1,510 households, the number of low-income households is 220 , the number of medium-income households is 739 , and the number of high-income households is 551 . Neighborhood types (ntypela to ntype8) and income level (inc_a 11 ) are dummy variables, and others are continuous variables. ntypela comprises $24 \%$ of Mecklenburg County, ntype3 comprises $37 \%$, ntype4 $31 \%$, ntype5 24\%, ntype6 $21 \%$, ntype7 $16 \%$, and ntype $823 \%$. These neighborhood types are not mutually exclusive, so 
each TAZ can have more than one 5 The mean log-sum of access to each subcenter (each_acc1 to each_acc10) ranges from -78 to 0 . Because travel is a disamenity, the log-sum is a negative value and the maximum value is close to zero. Values close to zero indicate more opportunities for jobs and other activities close to the residential location, and larger negative values indicate that the residential location has lower access to opportunities. This measurement also applies to access to the closest subcenter among 10 subcenters from a particular TAZ (min_acc), and the cumulative access to all 10 subcenters from a particular TAZ (sum_acc).

For walkability and local accessibility factors, the accumulated standardized scores multiplied by the corresponding factor loadings are used to calculate the mean factor score. Most of the indicators of local accessibility are measured by distance, so a negative value for local accessibility is expected. In this way, we expect that the probability of choosing a particular TAZ decreases as its distance to local land uses increases. On the other hand, a home affordability variable (mpv_inc) is measured by the ratio of mean TAZ-level home price to individual household income, the average of which is 6.15-greater than the home price multiplier of 3.65 used as the maximum threshold of home affordability in sampling choices.

Since the heteroscedastic models perform better than the conditional logit models ${ }^{6}$ our results focus exclusively on the heteroscedastic logit models. Among these, the models with sampled choices have much lower Bayesian Information Criterion (BIC) 7 values than those with the full choice set for all income groups. This indicates that the models with sampled choices fit the data better than those with the full choice set. This is partly because the models with a reduced choice set could account for the correlation (spatial or non-spatial) that is introduced by considering the universal choice set of all TAZs. The three access models are consistent in terms of directionality of the mean effect for significant variables. For these reasons, in this paper, we exclusively show the results of the heteroscedastic logit models with sampled choices.

${ }^{5}$ Since neighborhood types are not mutually exclusive, the coefficients of these variables may be biased. However, since our study regarded neighborhood types as control variables, we generously accepted these biased estimates. Our future work will correct this issue.

${ }^{6}$ All conditional logit models converged satisfactorily with McFadden's adjusted $\mathrm{R}^{2}$ (adjusted rhosquare) values between 0.018 and 0.38 . for all income groups, McFadden's adjusted $\mathrm{R}^{2}$ values are much higher for conditional logit models with sampled choices and with access to each individual subcenter than they are for other models. McFadden's adjusted $\mathrm{R}^{2}$ is meaningless in the heteroscadestic models where the number of degrees of freedom is less than the number of parameters. Thus, we used the Bayesian Information Criterion (BIC) to compare the conditional logit models to the heteroscedastic logit models. We found that the heteroscedastic logit models were consistently more favorable than the conditional logit models with much lower BIC values. This suggests that accounting for unobserved response heterogeneity across households provides additional explanatory power.

${ }^{7} B I C_{i}=-2 l_{i}+k_{i} \ln n$ is the maximum $\log$-likelihood for $i, k_{i}$ is the number of free parameters for $i$, and $n$ is the number of observations. 
Additionally, by comparing BIC values for the heteroscedastic models with sampling, we found that models that including access to each subcenter have the best fit, even though the BIC values tend to penalize heavily the more complex models. Furthermore, models with cumulative access to all subcenters fit better than models with access to the closest subcenter. This is strong evidence that modeling each subcenter independently should be the preferred approach when attempting to explain residential location decisions.

Table 2: Descriptive statistics of variables.

\begin{tabular}{|c|c|c|c|c|c|c|}
\hline Variable & Definition & Obs & Mean & SD & Min & Max \\
\hline \multicolumn{7}{|c|}{ TAZ characteristics: } \\
\hline highincac & High income acres & 1024 & 73.37 & 121.98 & 0 & 953.49 \\
\hline per_black & $\%$ black residents (BLACK/POP) & 1024 & 8.646 & 16.092 & 0 & 100 \\
\hline ntypela & $\begin{array}{l}\text { Core } \mathrm{CBD} \& \mathrm{CBD} \text { with residence } \\
\text { ntype1 \& ntype2 }\end{array}$ & 1024 & 0.24 & 0.43 & 0 & 1 \\
\hline ntype3 & First ring of suburbs & 1024 & 0.47 & 0.50 & 0 & 1 \\
\hline ntype4 & Second ring of suburbs & 1024 & 0.31 & 0.46 & 0 & 1 \\
\hline ntype5 & $\begin{array}{l}\text { Suburban single family residences with } \\
\text { some commercial and transit services }\end{array}$ & 1024 & 0.24 & 0.43 & 0 & 1 \\
\hline ntype6 & Suburbs with low regional access & 1024 & 0.21 & 0.40 & 0 & 1 \\
\hline ntype7 & $\begin{array}{l}\text { Exurbs with low regional access (Refer- } \\
\text { ence) }\end{array}$ & 1024 & 0.16 & 0.37 & 0 & 1 \\
\hline ntype8 & $\begin{array}{l}\text { Isolated residences in forested, indus- } \\
\text { trial, and commercial areas }\end{array}$ & 1024 & 0.23 & 0.42 & 0 & 1 \\
\hline each_acc1 & Minimum access to subcenter 1 & 1024 & -39.74 & 13.06 & -69.77 & 0.00 \\
\hline each_acc2 & Minimum access to subcenter 2 & 1024 & -34.05 & 11.09 & -64.46 & 0.00 \\
\hline each_acc3 & Minimum access to subcenter 3 & 1024 & -21.42 & 11.64 & -60.39 & 0.00 \\
\hline each_acc4 & Minimum access to subcenter 4 & 1024 & -31.01 & 11.89 & -66.87 & 0.00 \\
\hline each_acc5 & Minimum access to subcenter 5 & 1024 & -30.37 & 12.51 & -70.23 & 0.00 \\
\hline each_acc6 & Minimum access to subcenter 6 & 1024 & -29.52 & 12.42 & -69.67 & 0.00 \\
\hline each_acc7 & Minimum access to subcenter 7 & 1024 & -34.38 & 13.18 & -74.10 & 0.00 \\
\hline each_acc8 & Minimum access to subcenter 8 & 1024 & -36.74 & 13.54 & -77.45 & 0.00 \\
\hline each_acc9 & Minimum access to subcenter 9 & 1024 & -38.12 & 13.10 & -75.08 & 0.00 \\
\hline each_acc10 & Minimum access to subcenter 10 & 1024 & -26.84 & 12.02 & -62.56 & 0.00 \\
\hline min_acc & Access to closest subcenter & 1024 & -48.23 & 9.72 & -77.45 & -30.07 \\
\hline sum_acc & Cumulative access to each subcenter & 1024 & -322.19 & 82.67 & -651.90 & -214.82 \\
\hline walkability & Derived factor & 1024 & 0.63 & 1.46 & -1.32 & 8.32 \\
\hline accessibility & Derived factor & 1024 & -0.001 & 1.20 & -3.44 & 5.02 \\
\hline \multicolumn{7}{|c|}{ Interaction of TAZ and household characteristics: } \\
\hline mpv_inc & Mean housing price / household income & 1510 & 6.15 & 17.64 & 0.01 & 241.90 \\
\hline \multicolumn{7}{|c|}{ Household characteristics: } \\
\hline children & Number of children & 1510 & 0.19 & 0.50 & 0 & 5 \\
\hline inc_al 1 & Income levels (11 categories) & 1510 & 6.31 & 2.49 & 1 & 11 \\
\hline numveh & Number of vehicles & 1510 & 1.83 & 0.88 & 0 & 10 \\
\hline workers & Number of workers & 1510 & 1.14 & 0.81 & 0 & 5 \\
\hline
\end{tabular}




\subsection{All households}

Table 3 summarizes the results of the heteroscedastic logit model with sampled choices and three access specifications for all households. In each access model, the number of children in the household, household income, and the number of workers in the household significantly contribute to the observed heterogeneous distribution of tastes across households. Controlling this observed heteroscedasticity, we found that households are more likely to choose areas with more high-income household acres and with a lower percentage of black residents. The coefficient of home affordability is positive, suggesting that households are likely to spend their maximum budget to live in areas within their budget constraints. Furthermore, built-environment variables including neighborhood type and local accessibility are significant in the three access models. Our results support the long-tested finding that socio-demographic and neighborhood attributes are strong determinants of residential location decisions.

Households appear more likely to live close to subcenter 5, and less likely to live close to subcenters 4 and 8 (see Figure 2). These significant subcenters exhibit similar specializations in spatially dispersed business sectors, such as financial services, various other services, and transport/warehouse/utilities. We did expect to see different impacts of accessibility to workplaces by industry sector discovered by other studies (Dubin, 1991; Crane and Chatman, 2003), although an examination of colinearity suggests that the measures of access to each subcenter are very highly correlated. However, we found that many of the insignificant results for the model in the first column are a result of the high colinearity.

Given the high colinearity, it is not surprising that models including variable terms for access to the closest subcenter (min_acc) and cumulative access to all subcenters (sum_acc) show less significant impacts than those with access to individual subcenter. Access to the closest subcenter is statistically significant but has an unexpected sign, and cumulative access to all subcenters is not statistically significant. This suggests that improving access to the closest subcenter and cumulative access to all subcenters will not necessarily yield desirable results in terms of residential self-sorting to capitalize on such access.

\subsection{High-income households}

Numerous studies have shown that differences in income imply differences in locational determinants (Dubin 1991; Deitz 1998; Levine 1998; Bhat \& Guo 2004; Cho et al. 2005). Indeed, re-estimating the models by household income group revealed remarkable differences among income groups. For high-income households, the heteroscedastic logit model produced a pattern of results similar to those produced by the model of all households, as illustrated in Table 4. Among household attributes, household income and number of motorized vehicles emerged as significant causes of taste 


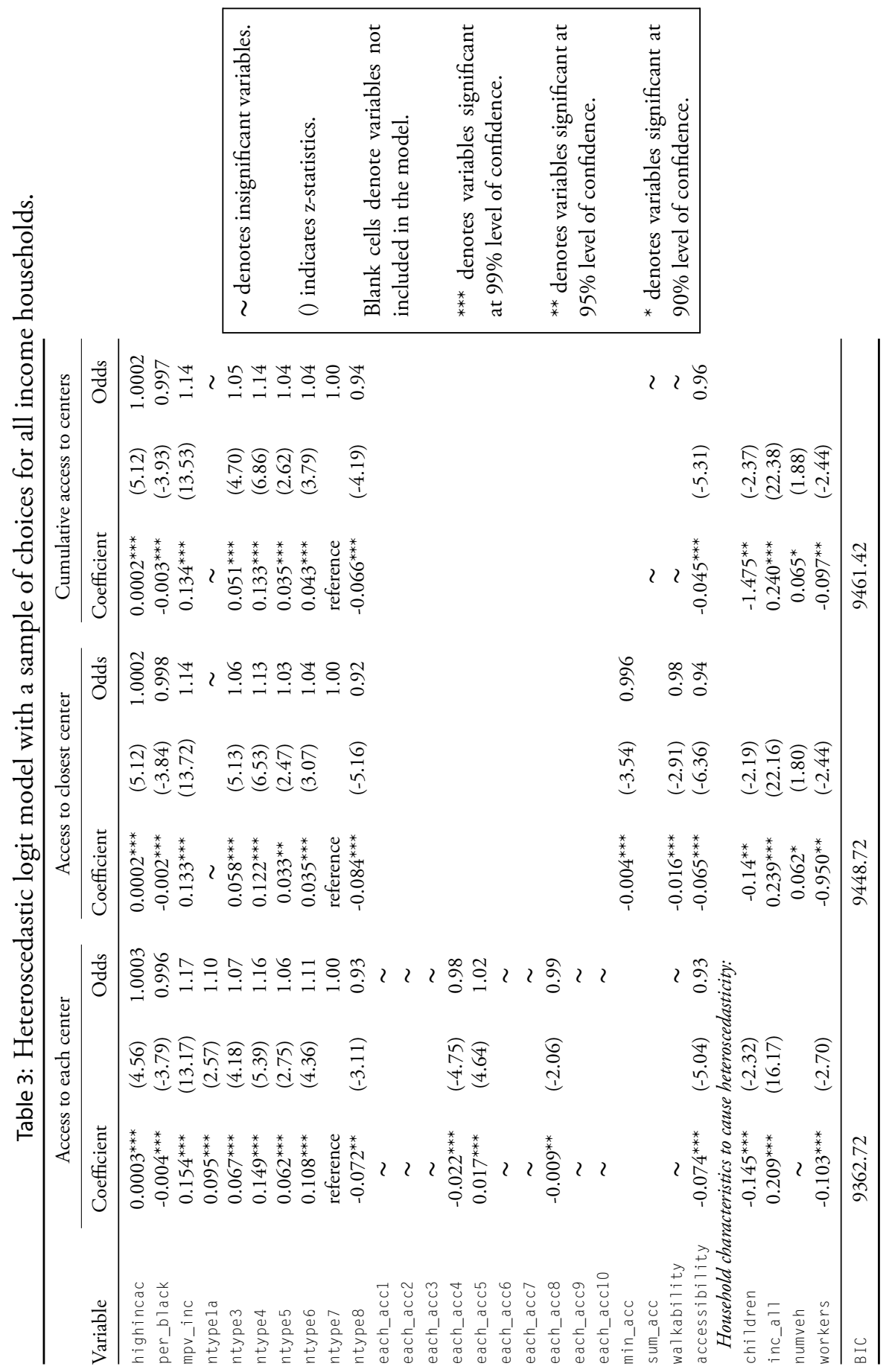


variation affecting the residential location decisions of high-income households.

By controlling for significant household and neighborhood attributes, we found that the results from the two simple ways of specifying access to the closest subcenter and cumulative access to all subcenters are not statistically significant. These results imply that high-income households may take into consideration access to certain individual subcenters in their residential location decisions, and that they appear to ignore other subcenters. Again the access measures to individual subcenters have very high colinearity.

\subsection{Medium-income households}

As illustrated in Table 5, medium-income households' preferences and tastes with regard to residential location choice differ from those of high-income households. Medium-income households' tastes in location even vary based on household characteristics, such as number of children, income, and number of motorized vehicles. We found that access to the closest subcenter does not significantly influence residential location choice, but that cumulative access to all subcenters is a significant determinant. Plaut (2006) found that two workers in a household increase or decrease their commuting distances together, treating their workplaces as complements. In the same way, our results are consistent with the complementary treatment of subcenters.

However, the specification that includes access to individual subcenters provides a more nuanced interpretation of their impact on location choices, even though colinearity remains a concern. Specifically, medium-income households are more likely to live close to subcenters 2 and 7 , and are less likely to live close to subcenters 3 and 8 . This indicates that they have strong preferences for certain subcenters in their residential location decisions, as expected. Subcenter 2 is located at a well-known research park in the Charlotte area that specializes in business sectors such as the arts, entertainment, recreation, accommodations, food service, public administration, and various other services and transport/warehouses/utilities. The well-paying jobs and the service opportunities found in subcenter 2 may attract medium-income households to the area. On the other hand, subcenters 3, 7 and 8 include similar specialized business sectors: professional and financial services, various other services and transport/warehouses/utilities. Even with these similarly specialized sectors, medium-income households prefer to live close to subcenter 7 and avoid living close to subcenter 8 . This pattern may be explained based on the existing land use pattern: subcenter 7, which is located in Charlotte, contains more single-family residences than subcenter 8 , which is located in the city of Matthews. Also, medium-income households are less likely to live close to subcenter 3 (located in the $\mathrm{CBD}$ ) and its residential areas than in other areas. 


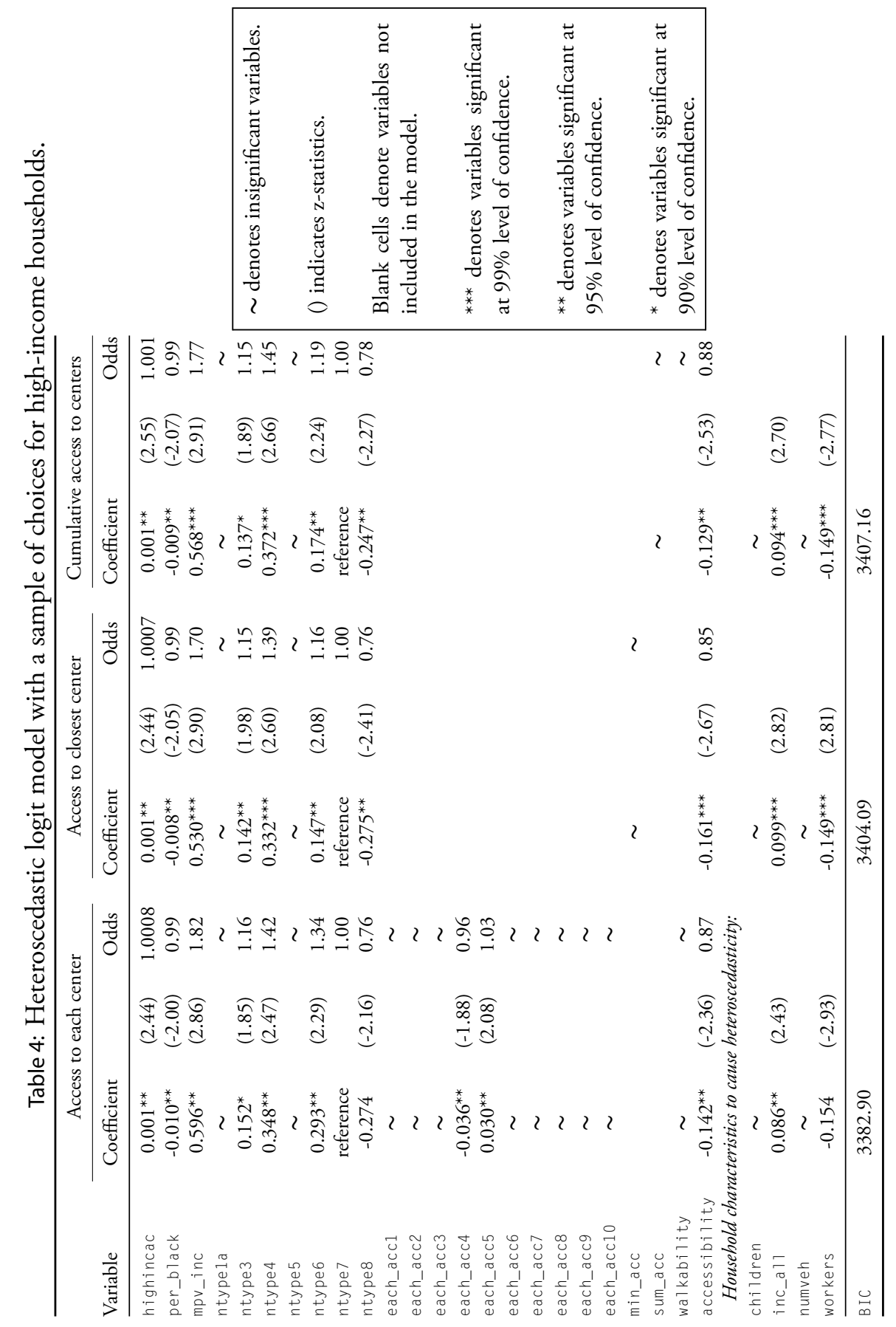




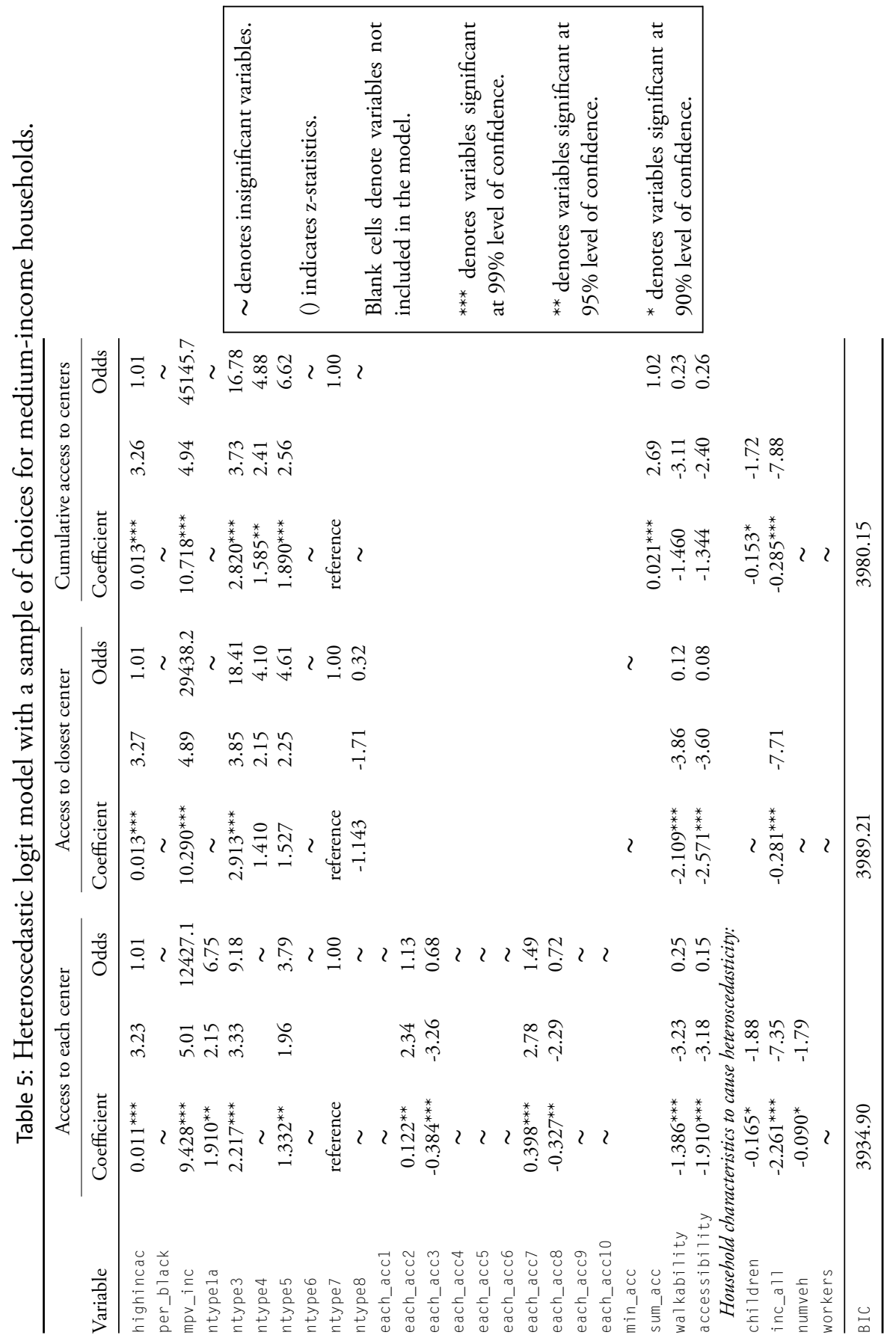




\subsection{Low-income households}

For low-income households, income, number of workers, and number of motorized vehicles cause unobserved response heterogeneity across households in the models, as shown in Table 6 . When controlling for household variation in tastes and neighborhood attributes, we found that both the measure of access relying on proximity to the closest subcenter and the cumulative measure of access to all subcenters, are significant determinants of residential location choice, with expected signs. This implies that low-income households are more likely to choose their residence closer to the closest subcenter, while they are also likely to live at locations which are close to all the subcenters at the same time.

In addition, the detailed specification that includes each subcenter also has high colinearity, indicating that low-income households are more likely to live close to subcenters specializing in transportation, utilities and warehouses (such as subcenter 9). Meanwhile, as discussed above, subcenter 5, with its upscale mixed uses and large shopping mall, may even attract low-income households, due to the presence of current and potential workplaces and the availability of transit systems.

\section{Conclusions}

This research focused on identifying the role that employment subcenters play in influencing residential location decisions. For many people, employment and activity subcenters embody not only current opportunities for jobs and recreation, but also future such opportunities. This observation led to the hypothesis that households are more likely to select areas with improved accessibility to employment subcenters, and further that the economic specialization of particular subcenters may have an impact on household location decisions.

We found a consistent association between access to subcenters and residential location choice. Our results suggest that some of the individual employment subcenters are important determinants driving residential location decisions for the three household income groups. Furthermore, models stratified by household income group suggest distinct residential location preferences relative to specific individual employment subcenters. These patterns can be explained not only by existing land use and transportation patterns, but also by subcenter economic specialization, although the subcenter variables exhibited strong multicolinearity which may explain why some subcenters were not statistically significant.

As shown by our conceptualization of accessibility to employment subcenters (access to each subcenter, access to the closest subcenter, and cumulative access to all subcenters), previous research has been limited in its ability to capture the impacts of regional accessibility in polycentric cities. It is not enough to examine access to a single 


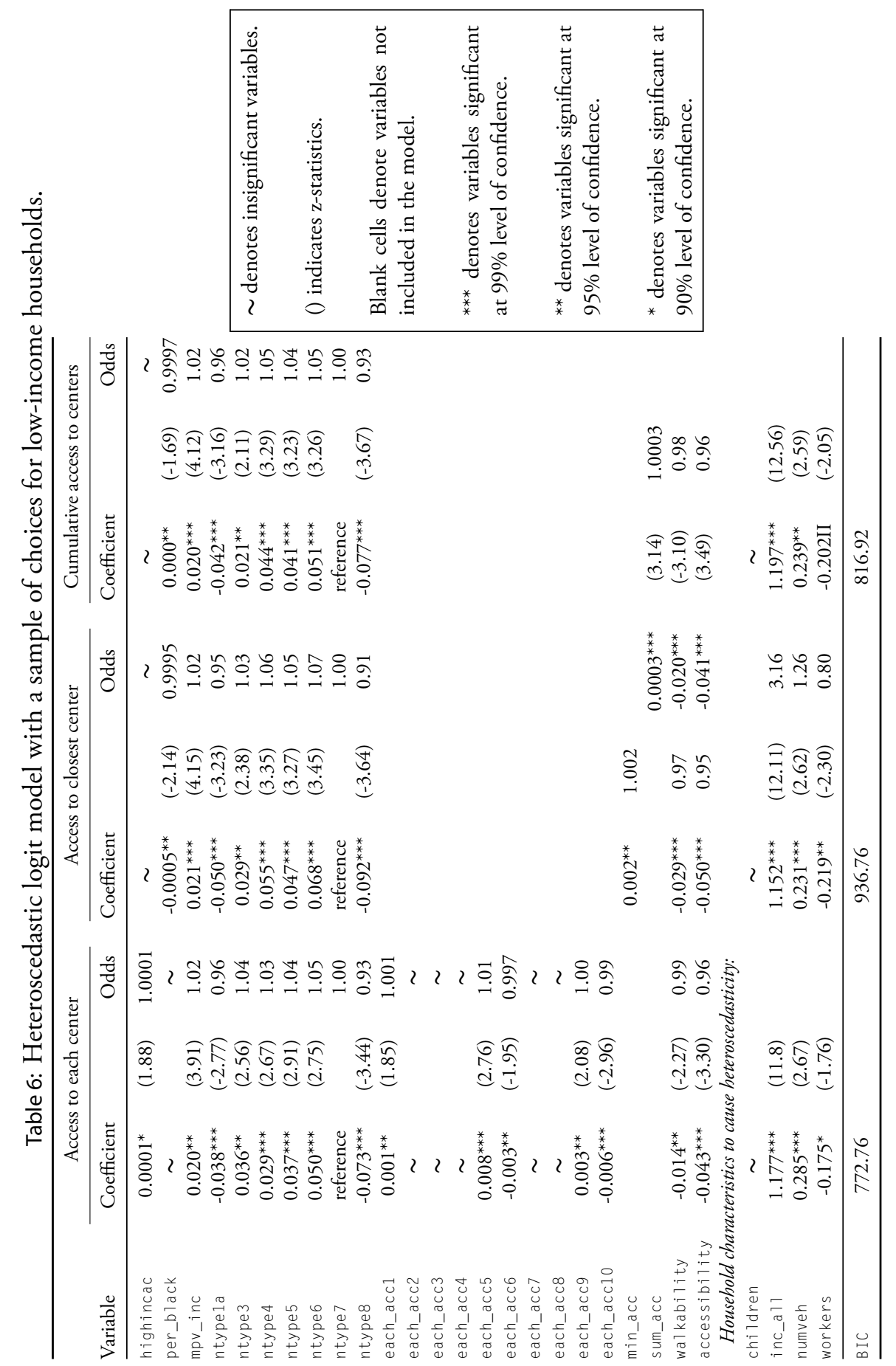


or most proximate center. Overall our findings suggest that employment subcenters interact in complex ways in influencing location choices. In some cases the evidence suggests that subcenters may substitute for each other, whereas in other cases they complement functions of other subcenters, creating synergies and interactions that location decision-makers view jointly beneficial. Yet, the disaggregate treatment of subcenters yielded models with better fit than models that attempted to examine proximity to the closest subcenter or a single, additive measure of accessibility to all subcenters. This suggests that measuring opportunities in space discretely is a promising strategy for understanding location decisions.

This study has several limitations. First, because it used cross-sectional data, it could not capture dynamic changes of the housing market over time and their influences on residential location choice. Second, the unique characteristics of the study area prevent generalization of our findings. Third, the effect of accessibility to subcenters on residential location choice was estimated for all aggregated households regardless of number of workers in the household; future work will examine the influence of accessibility to subcenters on residential location choice for households with and without workers separately, and then compare it with the influence of access to the current workplace on residential location for worker-households. Similarly workplace location choices (for workers) were assumed to be endogenous; in reality, for some workers, job sites are exogenously determined. Including distance to current work (for workers) may enhance the explanatory power of the model, and could be used to test whether current employees are balancing the commuting requirements of their current jobs against the potential commuting requirements of future jobs. In the long term, we will examine the influence of subcenters using longitudinal data and employ other study areas.

A fourth limitation of the present research is that subcenters are defined a priori, with little regard for the spatial dispersion of activity and employment opportunities, and using criteria that are somewhat arbitrary. Although, by definition, subcenters have high concentrations of jobs, most jobs and destinations in Mecklenburg County are spatially dispersed. A comparison of our model against models with measures of access to all employment, regardless of its concentration, would provide additional insights into the benefits of high accessibility to employment. Despite these limitations, we believe that this research has contributed to an understanding of the role of accessibility to employment subcenters in influencing residential location decisions, and of the consequences and potential effectiveness of planning interventions to improve regional access. 


\section{Acknowledgements}

The authors gratefully acknowledge the support received through EPA Star Grant RD83183501-0 in conducting this work, as well as the helpful feedback given by reviewers and by participants in the 2007 Access to Destinations Conference at the University of Minnesota. All errors and omissions remain ours.

\section{References}

Abraham, J.E., and J.D. Hunt 1997. Specification and estimation of nested logit model of home, workplaces, and commuter mode choices by multiple-worker households.Transportation Research Record, 1997; 1606: 17-23.

Allenby G.M., and J.L. Ginter 1995. The effects of in-store displays and feature advertising on consideration sets.International Journal of Research in Marketing 12: 67-80.

Anas, A. 1981. The estimation of multinomial logit models of joint location and travel mode choice from aggregated data. Journal of Regional Science 21 (2): 223-242.

1982. Residential location markets and urban transportation: Economic theory, econometrics, and policy analysis with discrete models. New York: Academic Press.

1995. Capitalization of urban travel improvements into residential and commercial real estate: simulations with a unified model of housing, travel mode and shopping choices. Journal of Regional Science 35: 351-375.

Anas, A., R. Arnott, and K.A. Small 1998. Urban spatial structure. Journal of Economic Literature 36: 1426-1464.

Axhausen, K.W. 2007. Accessibilities: Long-term perspectives. Presented at the International Conference "Access to Destinations" at University of Minnesota, Minneapolis, MN, August 2007.

Baradaran, S., and F. Ramjerdi 2001. Performance of accessibility measures in Europe. Journal of Transportation Statistics, 4: (2/3), 31-48.

Beckmann, M., and Y. Papageorgiou 1989. Heterogeneous tastes and residential location. Journal of Regional Science 29 (3): 217-323.

Ben-Akiva, M., and S.R. Lerman 1985. Discrete choice analysis: theory and application to travel demand. Cambridge, MA: The MIT Press.

Ben-Akiva, M., and J.L. Bowman 1998. Integration of an activity-bases model system and a residential location model. Urban Studies 35 (7): 1131-1153.

Berechman, J., and R. Paaswell 2001. Accessibility improvements and local employment: an empirical analysis. Journal of Transportation and Statistics 4 (2/3): 1-17.

Bhat, C.R. 1995. A heteroscedastic extreme value model of intercity travel mode choice. Transportation Research Part B 29 (6): 471-483. 
Bhat, C.R., and J. Guo 2004. A mixed spatially correlated logit model: formulation and application to residential choice modeling. Transportation Research B 38: $147-168$.

2007. A comprehensive analysis of built environment characteristics on household residential location choice and auto ownership levels. Transportation Research B 41 (5): 506-526.

Berke, P., D. Godschalk, E. Kaiser, and D.A. Rodriguez 2006. Urban Land Use Planning. 5 ed. Urbana-Champaign: University of Illinois Press.

Cervero, R., and K.-L. Wu 1997. Polycentrism, commuting, and residential location in the San Francisco Bay Area. Environment and Planning A 29: 865-886.

Cervero, R. 2002. Built environments and mode choice: towards a normative framework. Transportation Research D 7, 265-284.

Cho, S., D.N. Newman, and D.N. Wear 2005. Community choices and housing demands: a spatial analysis of the Southern Appalachian Highlands. Housing Studies 20: 549-569.

Clark, W.A.V., Y. Huang, and S. Withers 2003. Does commuting distance matter? Commuting tolerance and residential change. Regional Science and Urban Economics 33: 199-221.

Craig, S.G., and P.T. Ng 2001. Using quantile smoothing splines to identify employment subcenters in an multicentric urban area. Journal of Urban Economics 49: $100-120$.

Crane, R. 1996. The influence of uncertain job location on urban from and the journey to work. Journal of Urban Economics 39: 342-356.

Crane, R., and D. Chatman 2003. Traffic and sprawl: evidence from U.S. commuting, 1985 to 1987. Planning and Markets 6 (1): 1-17.

DeShazo, J.R., and G. Fermo 2002. Designing choice sets for stated preference methods: the effects of complexity on choice consistency. Journal of Environmental Economics and Management 44: 123-143.

Dietz, R. 1998. A joint model of residential and employment location in urban areas. Journal of Urban Economics 44 (2): 197-215.

Dubin, R. 1991. Commuting patterns and firm decentralization. Land Economics 67 (1): 15-29.

El-Geneidy, A.M., and D.M. Levinson 2006. Access to destinations: Development of accessibility measures. Report \#1 in the series Access to Destinations Study. Report\#2006-16. Web access: http://conservancy.umn.edu/bitstream/638/1/200616.pdf.

Gordon, P., H.W. Richardson, and M. Jun 1991. The commuting paradox: evidence from the top twenty. Journal of the American Planning Association 57: 416-420.

Giuliano, G., and K.A. Small 1991. Subcenters in the Los Angeles region. Regional Science and Urban Economics 21: 163-182. 
1993. Is the journey to work explained by urban structure? Urban Studies 30 (9): 1485-1502.

Glaeser, E.L., and M.E. Kahn 2001. Decentralized employment and the transformation of the American city. NBER Working Paper No. 8117. National Bureau of Economic Research, Cambridge, MA.

Hamilton, B. 1983. Wasteful commuting. Journal of Political Economy 90: 1035-1053.

Handy, S.L. and K. Clifton (2001). Evaluating neighborhood accessibility: Possibilities and practicalities. Journal of Transportation and Statistics 4 (2/3): 67-78.

Hensher, D., J. Louviere, and J. Swait 1999. Combining sources of preference data. Journal of Econometrics 89: 197-221.

Hole, A.R. 2006. Small-sample properties of tests for heteroscedasticity in the conditional logit model. Health, Econometrics and Data Group Working Paper. ISSN 1751-1976. Available at www.york.ac.uk/res/herc/pdfs/wp/06_04.pdf.

Lerman, S.R. 1975. A disaggregate behavioral model of urban mobility decisions. $\mathrm{PhD}$ dissertation, Massachusetts Institutes of Technology.

Levine, J.C. 1998. Rethinking accessibility and jobs-housing balancing. Journal of the American Planning Association 64 (2): 133-149.

Levinson, D.M. 1998. Accessibility and the journey to work. Journal of Transport Geography 6 (1): 11-21.

McCann, P. 2001. Urban and Regional Economics. New York, NY: Oxford University Press.

McDonald, J.F. 1987. The identification of urban employment subcenters. Journal of Urban Economics 21: 242-258.

McFadden, D. 1978. Modeling the choice of residential location. Transportation Research Record 672: 72-77.

McMillen, D.P. 2001. Nonparametric employment subcenter identification. Journal of Urban Economics 50: 448-473.

McMillen, D.P., and J.F. McDonald 1998. Suburban subcenters and employment density in Metropolitan Chicago. Journal of Urban Economics 43: 157-180.

Miller, H. 1999. GIS software for measuring space-time accessibility in transportation planning analysis. Paper presented at the International Workshop on Geographic Information Systems for Transportation and Intelligent Transportation Systems, Hong Kong.

Miller, E.J., J.D. Hunt, J.E. Abraham, and P.A. Salvini 2004. Microsimulating urban system. Computers, Environment and Urban Systems 28: 9-44.

Molin, E., and H.J.P. Timmermans 2003. Accessibility considerations in residential choice decisions: Accumulated evidence from the Benelux. Paper presented at the 2003 annual meeting of the Transportation Research Board, Washington, D.C.

Mok, D. 2007. Do two-earner households base their choice of residential location on both incomes? Urban Studies 44 (4): 723-750. 
Nechyba, T.J., and R.P. Strauss 1998. Community choice and local public services: A discrete choice approach. Regional Science and Urban Economics 28: 51-73.

Niemeier, D.A. 1997. Accessibility: An evaluation using consumer welfare. Transportation 24: 377-396.

Plaut, P. 2006. The intra-household choices regarding commuting and housing. Transportation Research A 40 (7): 561-571.

Rapaport, C. 1997. Housing Demand and Community Choice: An Empirical Analysis. Journal of Urban Economics 42 (2): 243-260.

Reginster, I., and F. Goffette-Nagot 2005. Urban environmental quality in two Belgian cities, evaluated on the basis of residential choices and GIS data. Environment and Planning A 37 (6): 1067-1090.

Rodriguez, D. 2004. Spatial choices and excess commuting: A case study of bank tellers in Bogotá, Colombia. Journal of Transport Geography 12 (1): 49-61.

Rodriguez, D., and J. Joo 2004. The relationship between non-motorized mode choice and the local physical environment. Transportation Research D 9 (2): 151-173.

Romani, J., J. Surinach, and M. Artiis 2003. Are commuting and residential mobility decisions simultaneous? The case of Catalonia, Spain. Regional Studies 37 (8): 813-826.

Sermons, M.W., and F.S. Koppelman 2001. Representing the differences between female and male commute behavior in residential location choice models. Journal of Transport Geography 9: 101-110.

Shen, Q. 1998. Spatial technologies, accessibility, and the social construction of urban space. Computers, Environment and Urban Systems 22 (5): 447-464.

Shen, Q. 2000. Spatial and Social Dimensions of Commuting. Journal of the American Planning Association 66 (1): 68-82.

Shukla, V., and P. Waddell 1991. Firm location and land use in discrete urban space: A study of the spatial structure of Dallas-Ft. Worth. Regional Science and Urban Economics 21: 225-253.

Small, K., and S. Song 1992. Wasteful commuting: A resolution. Journal of Political Economy 100 (4): 888-898.

Smith, S.S. 2004. Boom for whom? Education, desegregation, and development in Charlotte. State University of New York, Albany.

Timmermans, H.J.P. 2003. The saga of integrated land use-transport modeling: How many more dreams before we wake up? Paper read at 10th International Conference on Travel Behaviour Research, Lucerne, 2003.

Van Ommeren, J.N., P. Rietveld, and P. Nijkamp 1998. Spatial moving behavior of two-earner households. Journal of Regional Science 38 (1): 23-41.

Waddell, P. 1993. Exogenous workplace choice in residential location models: Is the assumption valid in a multimodal metropolis? Geographical Analysis 25 (1): $65-82$. 
1996. Accessibility and residential location: The interaction of workplace, residential mobility, tenure, and location choices. Presented at the 1996 Lincoln Land Institute TRED conference.

Walker, B., A. Marsh, M. Wardman, and P. Niner 2002. Modeling tenants' choices in the public rented sector: A stated preference approach. Urban Studies 39: 665-688.

Wu, Y.-H., and H. Miller 2002. Computational tools for measuring space-time accessibility within transportation networks with dynamic flow. Journal of Transportation and Statistics 4 (2/3): 1-14.

Zondag, B., and M. Pieters 2005. Influence of accessibility on residential location choice. Transportation Research Record 1902: 63-70. 